\section{SOI: 1.1 TAS DOI: $10.15863 /$ TAS}

International Scientific Journal Theoretical \& Applied Science

p-ISSN: 2308-4944 (print) $\quad$ e-ISSN: 2409-0085 (online)

Year: $2015 \quad$ Issue: 08 Volume: 28

Published: $30.08 .2015 \quad \underline{\text { http://T-Science.org }}$

SECTION 1. Theoretical research in mathematics.
Anna Olegovna Zheldasheva Senior Lecturer, Department of Differential Equations, Kabardino-Balkar State University H.M. Berbekova, Russia, Nalchik anna.zheldasheva@mail.ru

Vadim Nikolayevich Lesev Candidate of Physical and Mathematical Sciences, Associate Professor, Head of the differential equations of the Kabardino-Balkar State University H.M. Berbekova,

Russia, Nalchik diff@kbsu.ru

Liana Vladimirovna Dumaeva Candidate of Physical and Mathematical Sciences, Senior lecturer, Department of Differential Equations, Kabardino-Balkar State University H.M.

Berbekova, Russia, Nalchik armand97a@gmail.com

\title{
NONLOCAL BOUNDARY VALUE PROBLEMS FOR A DEGENERATE SECOND ORDER EQUATIONS WITH DISCONTINUOUS CONJUGATION CONDITIONS
}

\begin{abstract}
The work is devoted to the study of the unique solvability of nonlocal boundary value problems for a degenerate second-order equations with discontinuous transmission conditions. The question of the existence of the problem solutions is reduced to the equivalent of the Fredholm integral equation solvability and the uniqueness of the solution set based on the energy integrals method.

Key words: boundary value problem; the integral equation; the method of energy integrals.

Language: Russian

Citation: Zheldasheva AO, Lesev VN, Dumaeva LV (2015) NONLOCAL BOUNDARY VALUE PROBLEMS FOR A DEGENERATE SECOND ORDER EQUATIONS WITH DISCONTINUOUS CONJUGATION CONDITIONS. ISJ Theoretical \& Applied Science 08 (28): 76-79.

Soi: http://s-o-i.org/1.1/TAS-08-28-12 Doi: crossef http://dx.doi.org/10.15863/TAS.2015.08.28.12

\section{НЕЛОКАЛЬНАЯ КРАЕВАЯ ЗАДАЧА ДЛЯ ВЫРОЖДАЮЩЕГОСЯ УРАВНЕНИЯ ВТОРОГО ПОРЯДКА С РАЗРЫВНЫМИ УСЛОВИЯМИ СОПРЯЖЕНИЯ}

Аннотация: Работа посвящена исследованию однозначной разрешимости нелокальной краевой задачи для вырождающегося уравнения второго порядка с разрывнылми условиями сопряжения. Вопрос существования решения задачи эквивалентно редуцирован к вопросу разрешимости интегрального уравнения Фредгольма, а единственность решения установлена на основе метода интегралов энергии.

Ключевые слова: краевая задача; интегральное уравнение; метод интегралов энергии.
\end{abstract}

$$
\begin{aligned}
& \text { Рассмотрим уравнение } \\
& \operatorname{sgn} x u_{x x}+\operatorname{sgn} y u_{y y}-\lambda^{2} u=0,
\end{aligned}
$$

где $\lambda$ - произвольное действительное число, в конечной односвязной области $\Omega$, ограниченной жордановой кривой $\sigma$ с концами в точках $A(1,0)$, $B(0,1)$ расположенной в первом квадранте $x>0$, $y>0$ и характеристиками $B C: y-x=1, C D$ : $x+y=0, D A: x-y=1$ уравнения (1).

Пусть $\bar{J}_{1}=O A, \bar{J}_{2}=O B ; \Omega_{i}=\Omega \cap\{t<0\}-$ гиперболические части смешанной области $\Omega$, где $t \in \bar{J}_{i}, \quad i=1,2$, (при $i=1 \quad t=x$, а при $i=2$ $t=y) ; \quad \Omega_{0}=\Omega \bigcap\{x>0\} \cap\{y>0\} \quad$ - эллиптическая часть области $\Omega$.

Под регулярным решением уравнения (1) в области $\Omega$, будем понимать функцию $u(x, y)$ из класса $C\left(\bar{\Omega}_{j}\right) \cap C^{1}\left(\Omega_{1} \cup J_{1}\right) \cap C^{1}\left(\Omega_{2} \cup J_{2}\right) \cap$ $\cap C^{1}\left(\Omega_{0} \cup J_{1} \cup J_{2}\right) \cap C^{2}\left(\Omega \backslash\left(C D \cup J_{1} \cup J_{2}\right)\right), \quad j=\overline{0,2}$ удовлетворяющую уравнению (1) и такую, что частные производные $u_{y}(x, 0), u_{x}(0, y)$ могут обращаться в бесконечность порядка меньше единицы на концах интервалов $\bar{J}_{i}$. Предположим, что кривая $\sigma$ удовлетворяет 
условию Ляпунова и оканчивается дугами окружности $\sigma_{0}: x^{2}+y^{2}=1$ сколь угодно малой длины.

Задача S. Найти регулярное в области $\Omega$ решение $u(x, y)$ уравнения (1), удовлетворяющее краевым условиям

$$
\begin{gathered}
a(s) \frac{\partial u}{\partial n}+\left.b(s) u\right|_{(x, y) \in \sigma}=\varphi(s), \quad s \in[0, l], \\
\tilde{A}_{1 x}^{0}\left\{u\left[\theta_{1}(x)\right]\right\}+c_{1}(x) u(x, 0)=d_{1}(x), \\
\tilde{A}_{1 y}^{0}\left\{u\left[\theta_{2}(y)\right]\right\}+c_{2}(y) u(0, y)=d_{2}(y)
\end{gathered}
$$

и условиям сопряжения

$$
\begin{gathered}
\tau_{1}^{-}(x)=\alpha_{1}(x) \tau_{1}^{+}(x)+\gamma_{1}(x), \\
v_{1}^{-}(x)=\beta_{1}(x) v_{1}^{+}(x)+\delta_{1}(x) \tau_{1}^{+}(x)+\sigma_{1}(x), \quad \forall x \in \bar{J}_{1}, \\
\tau_{2}^{-}(y)=\alpha_{2}(y) \tau_{2}^{+}(y)+\gamma_{2}(y), \\
v_{2}^{-}(y)=\beta_{2}(y) v_{2}^{+}(y)+\delta_{2}(y) \tau_{2}^{+}(y)+\sigma_{2}(y), \quad \forall y \in \bar{J}_{2} .
\end{gathered}
$$

Здесь $\theta_{1}(x)=\frac{x+1}{2}+i \frac{x-1}{2}, \quad \theta_{2}(y)=\frac{y-1}{2}+i \frac{y+1}{2}-$ аффиксы точек пересечения характеристик, выходящих из точек $(x, 0) \in \bar{J}_{1}, \quad(0, y) \in \bar{J}_{2} \quad$ с отрезками характеристик $A D$ и $B C ; s$ - длина дуги кривой $\sigma$ отсчитываемая от точки $A ; n$ внешняя нормаль;

$$
\tilde{A}_{1 t}^{0}[f(t)]=f(t)-\int_{1}^{t} f(\xi) \frac{\partial}{\partial \xi} I_{0}(|\lambda| \sqrt{(t-1)(t-\xi)}) d \xi,
$$$$
\varphi(s), a(s), b(s) \in C[0, l], \quad d_{i}(t) \in C\left(J_{i}\right) \cap C^{\left(2, h_{i}\right)}\left(J_{i}\right),
$$
$\delta_{i}(t), \sigma_{i}(t) \in C\left(\bar{J}_{i}\right) \cap C^{\left(2, h_{i}\right)}\left(J_{i}\right), \alpha_{i}(t), \quad \beta_{i}(t), \gamma_{i}(t)$, $c_{i}(t) \in C^{1}\left(\bar{J}_{i}\right) \cap C^{\left(2, h_{i}\right)}\left(J_{i}\right)$ - заданные функции, причем $\quad \beta_{i}^{2}(t)+\alpha_{i}^{2}(t)\left[1+2 c_{i}(t)\right]^{2} \neq 0$ $a^{2}(s)+b^{2}(s) \neq 0, \quad$ и $\quad \alpha_{i}(t) \beta_{i}(t)\left[1+2 c_{i}(t)\right] \neq 0, \quad($ $t \in J_{i}, i=1,2$ ).

Кроме того, функции $d_{i}(t)$ могут обращаться в бесконечность порядка меньше единицы на концах интервалов $\bar{J}_{i}$.

Справедлива следующая

Теорема S. Если $a(s) b(s) \geq 0$, и $\bar{p}_{i}(t) \geq 0$, $\tilde{p}_{i}(0) \geq 0, \quad \tilde{p}_{i}^{\prime}(t) \geq 0 \quad\left(\bar{p}_{i}(t) \leq 0, \quad \tilde{p}_{i}(0) \leq 0\right.$, $\left.\tilde{p}_{i}^{\prime}(t) \leq 0\right), \quad$ где $\quad \tilde{p}_{i}(t)=\frac{e^{2|\lambda| t}}{\alpha_{i}(t) \beta_{i}(t)\left[1+2 c_{i}(t)\right]}$, $\bar{p}_{i}(t)=-\beta_{i}(t) \delta_{i}(t), t \in \bar{J}_{i}, i=1,2$, то задача $\mathrm{S}$ не может иметь более одного решения.

Мы не будем останавливаться на доказательстве теоремы $\mathrm{S}$, которое проводится методами вспомогательных функций и интегралов энергии.

Докажем существование решения задачи $\mathrm{S}$.
Функциональные соотношения между $\tau_{i}^{-}(t)$ и $v_{i}^{-}(t)$, принесенные на единичные интервалы $J_{i}$, из гиперболических частей $\Omega_{i}$ смешанной области $\Omega$, имеют вид [1]

$$
\left[1+2 c_{i}(t)\right] \tau_{i}^{-}(t)=p_{i}(t)+\int_{t}^{1} v_{i}^{-}(\xi) I_{0}[|\lambda|(t-\xi)] d \xi,
$$

где $t \in \bar{J}_{i}, p_{i}(t)=2 d_{i}(t)-\tau_{i}^{-}(1) I_{0}[|\lambda|(1-t)], i=1,2$.

Рассмотрим вспомогательную задачу.

Задача N. Найти в области $\Omega_{0}$ регулярное решение $u(x, y)$ уравнения (1), удовлетворяющее условиям (2) и

$$
\begin{aligned}
& \left.\frac{\partial u}{\partial y}\right|_{y=0}=v_{1}^{+}(x), \quad x \in J_{1}, \\
& \left.\frac{\partial u}{\partial x}\right|_{x=0}=v_{2}^{+}(y), \quad y \in J_{2} .
\end{aligned}
$$

Как известно [2], при выполнении неравенства $a(s) b(s) \geq 0$, единственное решение задачи $\mathrm{N}$, имеет вид

$$
u(x, y)=-\int_{0}^{1} G(t, 0 ; x, y) \nu_{1}^{+}(t) d t-\int_{0}^{1} G(0, t ; x, y) \nu_{2}^{+}(t) d t+
$$

$$
+\int_{\sigma} \frac{\varphi(s)}{a(s)} G(\xi, \eta ; x, y) d s,
$$

где $G\left(x, y ; x_{0}, y_{0}\right)$ - функция Грина задачи N.

Переходя последовательно к пределам, при $y \rightarrow 0$ и $x \rightarrow 0$, из (6), находим функциональные соотношения между $\tau_{i}^{+}(t)$ и $v_{i}^{+}(t)$ принесенные из области $\Omega_{0}$ на $J_{i}$ в виде

$$
\begin{aligned}
& \tau_{1}^{+}(x)=-\int_{0}^{1} G(t, 0 ; x, 0) \nu_{1}^{+}(t) d t-\int_{0}^{1} G(0, t ; x, 0) \nu_{2}^{+}(t) d t+ \\
& +\int_{\sigma} \frac{\varphi(s)}{a(s)} G(\xi, \eta ; x, 0) d s, \\
& \tau_{2}^{+}(y)=-\int_{0}^{1} G(t, 0 ; 0, y) v_{1}^{+}(t) d t-\int_{0}^{1} G(0, t ; 0, y) v_{2}^{+}(t) d t+ \\
& +\int_{\sigma} \frac{\varphi(s)}{a(s)} G(\xi, \eta ; 0, y) d s .
\end{aligned}
$$

Исключая из последних двух равенств и соотношения (5) функции $\tau_{i}^{ \pm}(t)$, с учетом (3), (4), будем иметь

$$
A_{i}(t) v_{i}^{+}(t)+\frac{2 t}{\pi} \int_{0}^{1}\left[\frac{1}{\xi^{2}-t^{2}}-\frac{\xi^{2}}{1-\xi^{2} t^{2}}\right] v_{i}^{+}(\xi) d \xi-
$$




$$
\begin{gathered}
-\frac{2 t}{\pi} \int_{0}^{1}\left[\frac{1}{\xi^{2}+t^{2}}-\frac{\xi^{2}}{1+\xi^{2} t^{2}}\right] v_{j}^{+}(\xi) d \xi+ \\
+\frac{1}{\pi} \int_{0}^{1} K_{i i}(t, \xi) v_{i}^{+}(\xi) d \xi-\frac{1}{\pi} \int_{0}^{1} K_{i j}(t, \xi) v_{j}^{+}(\xi) d \xi+ \\
+\frac{\delta_{i}(t)}{\pi \beta_{i}(t)} A_{i}(t)\left[\int_{0}^{1} \ln \left|\frac{1-t^{2} \xi^{2}}{\xi^{2}-t^{2}}\right| v_{i}^{+}(\xi) d \xi+\right. \\
\left.+\int_{0}^{1} \ln \frac{1+t^{2} \xi^{2}}{\xi^{2}+t^{2}} v_{j}^{+}(\xi) d \xi\right]=\Phi_{i}(t),
\end{gathered}
$$

где $A_{i}(t)=-\frac{\beta_{i}(t)}{\left[1+2 c_{i}(t)\right] \alpha_{i}(t)}, K_{i j}(t, \xi),(i, j=1,2)$, $\Phi_{i}(t),(i=1,2)$ - известные функции [3], причем $K_{i j}(t, \xi), \quad(i \neq j)$ непрерывны в $0 \leq t, \xi \leq 1 \quad$ и принадлежат классу $C^{\left(1, h_{i}\right)}(0<t<1)$ при любом $t \in[0,1], \quad$ а ядро $K_{i i}(t, \xi)$ допускает оценку $\left|K_{i i}(t, \xi)\right|<M /|1-t \xi|^{\varepsilon_{i}}, \quad 0<\varepsilon_{i}<1, \quad i=1,2$. Кроме того, $\Phi_{i}(t) \in C^{\left(1, h_{i}\right)}\left(J_{i}\right), h_{i}>0, i=1,2$ и они могут иметь особенность порядка меньше единицы при $t \rightarrow 0$ и $t \rightarrow 1$.

Аналогично [4-7], система (7) путем замены неизвестных функций

$$
\mu_{1}(t)=v_{1}^{+}(t)+v_{2}^{+}(t), \quad \mu_{2}(t)=v_{1}^{+}(t)-v_{2}^{+}(t)
$$

и замены переменных

$$
\eta=\frac{2 t^{4}}{1+t^{8}}, \quad \tau=\frac{2 \xi^{4}}{1+\xi^{8}},
$$

приводится к виду

$$
A(t) \rho_{i}(\eta)+\frac{1}{\pi} \int_{0}^{1} \frac{\rho_{i}(\tau)}{\tau-\eta} d \tau=F_{i}(\eta),
$$

где

$$
\begin{gathered}
\rho_{1}(\eta)=t^{-3}\left(1+t^{8}\right) \mu_{1}(t), \\
\rho_{2}(\eta)=t^{-1}\left(1+t^{4}\right)^{-1}\left(1+t^{8}\right) \mu_{2}(t),
\end{gathered}
$$

а $F_{i}(\eta)$ - выражаются через $K_{i j}(t, \xi),(i, j=1,2)$ и другие известные функции, причем легко убедиться в том, что $F_{i}(\eta) \in C^{\left(1, h_{i}\right)}(0<\eta<1)$, $0<h_{i} \leq 1, i=1,2$ и они могут иметь особенность порядка меньше 1 , когда $\eta \rightarrow 0 \quad$ и $\eta \rightarrow 1$ соответственно.

В силу того, что $A^{2}(t)+1 \neq 0, \forall t \in \bar{J}_{i}, i=1,2$, система (8) является системой сингулярных интегральных уравнений нормального типа, а, следовательно, она может быть регуляризована. Используя известный способ регуляризации [8], получим систему интегральных уравнений Фредгольма второго рода со слабой особенностью. Для этой системы уравнений со свободными членами из класса $\left.C[0,1] \cap C^{1}\right] 0,1[$ справедливы теоремы Фредгольма $[9,10]$.

Из теоремы S, в силу эквивалентности (в смысле разрешимости) задачи $\mathrm{S}$ и полученной системы интегральных уравнений Фредгольма следует однозначная разрешимость последней. Определив искомые функции $v_{i}^{+}(t)$, находим $\tau_{i}^{ \pm}(t)$ и $v_{i}^{-}(t)$ из условий (3), (4).

Таким образом, решение задачи $\mathrm{S}$ в области $\Omega_{0}$ находим из (6), а в областях $\Omega_{1}, \Omega_{2}$ - как решение соответствующих задач Коши.

\section{References:}

1. Urinov AK (1983) On some boundary value problems for a mixed-type equation with nonsmooth degeneration lines // Proceedings of the Academy of Sciences of the Uzbek SSR Series Sci. Science, 1983. №2. pp. 29-34.

2. Kadyrov Z (1987) Boundary problem for equations of mixed type with a nonsmooth degeneration lines in an unbounded domain // Proceedings of the Academy of Sciences of the Uzbek SSR Series Sci. Sciences, 1987, №1. pp. 24-29.

3. Eleev VA, Lesev VN (2003) Problems with shift for degenerate hyperbolic equations and mixed. Lecture notes. - Nalchik Kab.-Balk. University Press, 2003. - 109 p.
4. Zainulabidov MM (1969) Some boundary value problems for equations of mixed type with two lines of degeneracy perpendicular // Differ. equation, 1969. T.5, №1. pp. 91-99.

5. Salakhitdinov MS (1974) The equations of mixed-composite type. - Tashkent: FAN, 1974. $-156 \mathrm{p}$.

6. Lesev VN (2003) A boundary value problem for the hyperbolic-elliptic second-order equations with perpendicular lines of degeneracy // Bulletin of Kabardino-Balkaria State University. A series of mathematical sciences. - Nalchik Kab.-Balk. University Press, 2003. - pp. 55-57.

7. Lesev VN (1998) The problem for the BitsadzeSamarskii equation elliptic-hyperbolic type with 


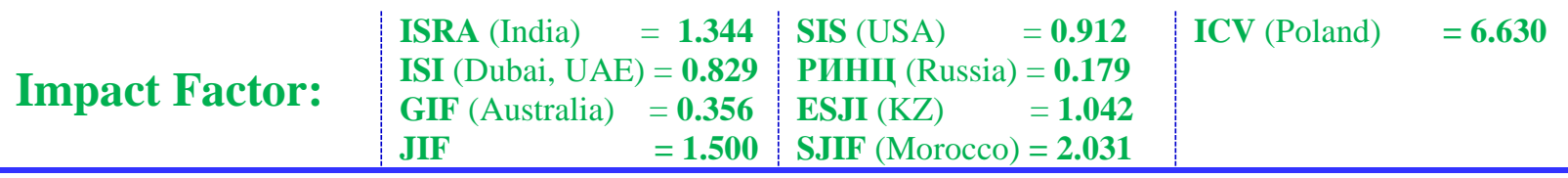

perpendicular lines of degeneracy // Abstracts of the North Caucasus regional scientific conference of students, graduate students and young scientists "Perspective-98." - Nalchik Kab.-Balk. University Press, 1998. - P. 33-35.8.

8. Muskhelishvili NI (1968) Singular integral equations. - Moscow: Nauka, 1968 - 512 p.

9. Zabreiko PP, et al. (1968) Integral equations. Moscow: Nauka, 1968. - 448 p.

10. Polyanin AD, Manzhirov AV (1998) Handbook of Integral Equations: Exact Solutions. Moscow: Factorial, 1998. - 384 p. 